\title{
Directed transport as a mechanism for protein folding in vivo
}

\author{
Ernesto González-Candela ${ }^{1}$ and V. Romero-Rochín ${ }^{2}$ \\ ${ }^{1}$ Universidad Autónoma Metropolitana - Iztapalapa. 09340 México, D.F. Mexico \\ 2 Instituto de Física, Universidad Nacional Autónoma de México, \\ Apdo. Postal 20-364, México D. F. 01000, Mexico.
}

(Dated: September 8, 2021)

\begin{abstract}
We propose a model for protein folding in vivo based on a Brownian-ratchet mechanism in the multidimensional energy landscape space. The device is able to produce directed transport taking advantage of the assumed intrinsic asymmetric properties of the proteins and employing the consumption of energy provided by an external source. Through such a directed transport phenomenon, the polypeptide finds the native state starting from any initial state in the energy landscape with great efficacy and robustness, even in the presence of different type of obstacles. This model solves Levinthal's paradox without requiring biased transition probabilities but at the expense of opening the system to an external field.
\end{abstract}




\section{INTRODUCTION}

Following the classical work of Anfinsen [1, 2] on protein folding, Levinthal [3] argued that if the protein multidimensional free energy landscape had a "golf course" like shape, with the hole being the native state, it would take essentially forever for a protein to fold correctly, provided the search were performed randomly. This is known as Levinthal's paradox, since proteins in vivo fold very fast and with high efficacy. However, Zwanzig et al [5, 6] demonstrated that, within a random search, a small bias for configurations closer to the native state would be enough to solve the paradox. Alternatively, to explain the efficacy of folding, the concept of a funneled energy landscape was developed [7, 8, 9, 10, 11, 12], with the further possibility of folding pathways. In such an scenario the process is driven mainly by free energy differences, that is, by "falling" through the funnel into the state of lowest free energy. This idea has been central in the study of protein folding. In this article, we propose a further alternative way out to the so-called Levinthal paradox, which does not necessarily needs a funnel structure nor introduces ad-hoc biased probabilities. We appeal to a Brownian motor or ratchet-like mechanism for the process of protein folding. This is a non-equilibrium process that requires, first, a free energy landscape with a ratchet-like asymmetry, and second, the consumption of energy from an external source [13]. We shall discuss below how these two essential ingredients may be justified.

The ratchet mechanism to produce directional current or motion has become a paradigm of the interplay of nonlinear phenomena with Brownian motion and unbiased external noise in mesoscopic systems [14, 15, 16, 17]. We shall use the term "ratchet" for any conservative mechanical system that has an ingrained spatial asymmetry; a simple one-dimensional example is a particle in a saw-tooth like potential. Due to the potentiality of these systems to describe mesoscopic systems such as biological ones [15, 16, 17, 18, , one should consider the ratchet to be immersed in a thermal bath. This adds dissipative and stochastic thermal forces that are related to each other through the fluctuation-dissipation theorem [19, 20]. As it was clearly pointed out by Feynman in his Lectures [21], the Second Law of Thermodynamics implies that a ratchet under the above conditions cannot generate current nor directional motion. Nevertheless, it is by now very well established that in order to obtain directional motion, the presence of an external unbiased time dependent force is necessary. This external force can be deterministic, a so-called "rocking" ratchet [14], or stochastic in 
nature [22]. In any case, any of those forces should be of zero average in time such that the current obtained is not a trivial consequence of the bias of those forces, but of their interplay with the ratchet potential. In the present article, since the external interaction of the protein during the folding process is supposed to yield energy through the consumption of high energy phosphate bonds, as adenosine-triphosphate (ATP) or guanosine-triphosphate (GTP), we assume an external, unbiased, stochastic force.

We shall show that, understanding by protein folding the search and finding of a target point or small section in the energy landscape, the ratchet mechanism is extremely effective and robust. As mentioned above, an interesting consequence of this process is that the landscape does not require a funnel structure [7, 10].

\section{THE MODEL}

The model consists of an overdamped Brownian "particle" moving in a multidimensional configuration space, the coordinates representing the effective degrees of freedom of a protein [24]. The environment enters as a dissipative thermal bath and the full folding process is driven by an unbiased external source.

Our central proposal is that the regularity and intrinsic asymmetries found in polypeptides, such as $\alpha$ helices and $\beta$ sheets, may arise from or give rise to an asymmetry at the level of the energy landscape. This feature is introduced through an asymmetric ratchet-like, conservative potential. The potential may be flat on average but it is provided with a small, deep and wide enough well, whose local minimum vicinity represents the native state of the protein. On top of the average potential there is a ratchet-like asymmetry that makes the potential towards the native state different than in the direction moving away from it. Figure 1 is a two-dimensional "free energy" landscape representation of the potential. Although the precise form of the landscape is essential for a given protein, here we shall use a very simple form for the configuration potential in order to show the virtues of the model. In $d$ dimensions the ratchet-like potential with a native state is given by,

$$
\begin{aligned}
V(\vec{x})= & V_{0}\left[\sin \left(\frac{2 \pi r}{\lambda}\right)+a \sin \left(\frac{4 \pi r}{\lambda}\right)+b \sin \left(\frac{6 \pi r}{\lambda}\right)\right] \\
& -V_{1} e^{-r^{2} / c}
\end{aligned}
$$

where $\vec{x}=x_{1}, x_{2}, \ldots, x_{d}$ are the configuration coordinates; $r=\left(x_{1}^{2}+x_{2}^{2}+\ldots+x_{d}^{2}\right)^{\frac{1}{2}}$; and $a$, 


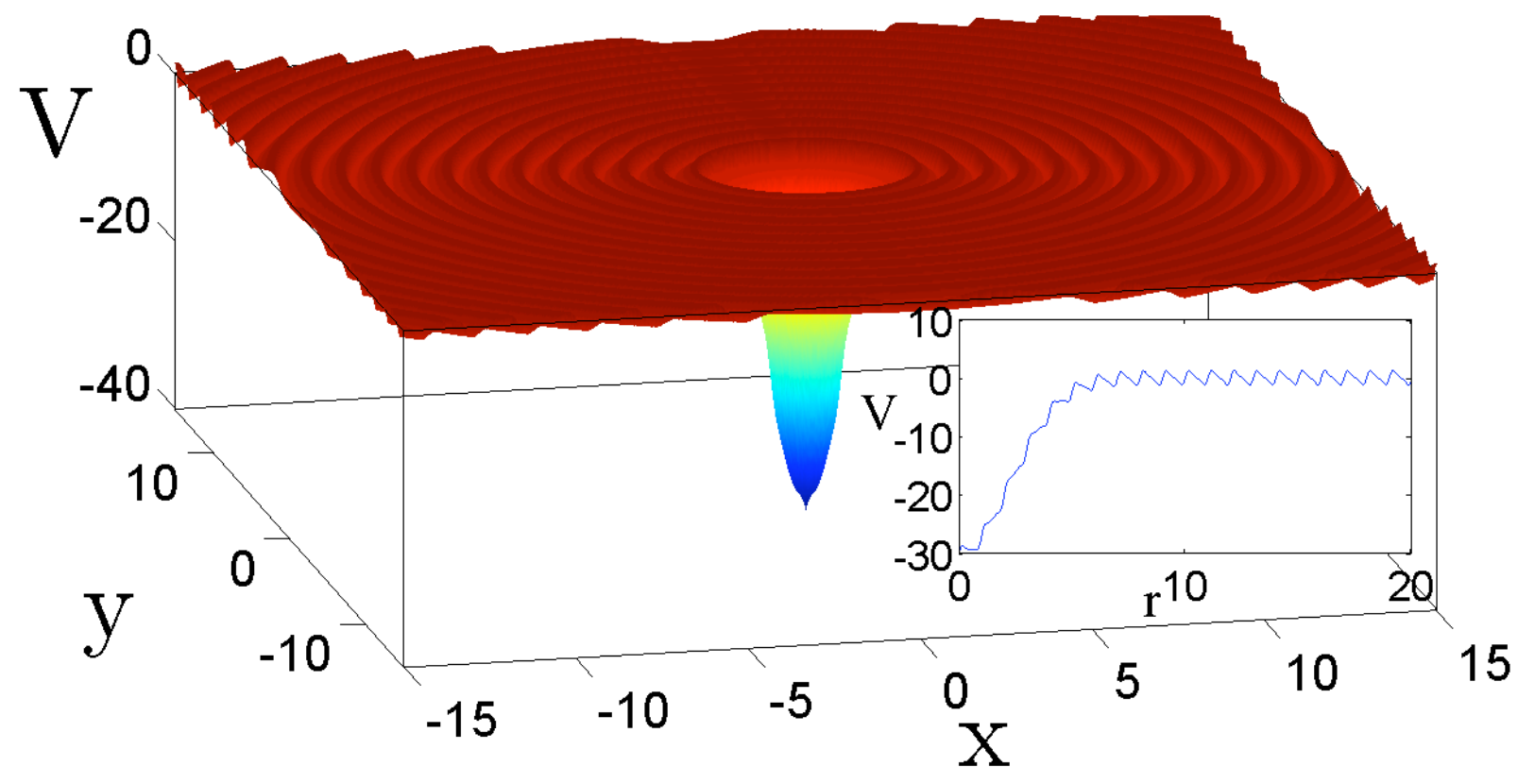

FIG. 1: (Color online) Two-dimensional representation of the protein "free energy" landscape, Eq.(1), with $\lambda=1, V_{0}=1, V_{1}=50, a=0.4, b=0.1$ and $c=10$. This landscape is flat on the average and has a ratchet-like asymmetry away and towards the native state, represented by a deep and wide enough minimum of the potential.

$b, c, V_{0}, V_{1}$ and $\lambda$ are positive real numbers that determine the structure of the potential. In the figure captions we specify their particular values. The native state is arbitrarily set at $r=0$ and it is a well given by the last term in $V(\vec{x})$. The first three terms give rise to a periodic ratchet potential with a radial asymmetry.

The above landscape potential can be make extremely more complicated and still work in the same way as the stylized model. That is, nonperiodic ratchets with wells, barriers or other structures can be introduced in order to approximate the potential to the real conditions of proteins, without affecting the essence of the model. This will be shown below.

Proteins are typically embedded in a viscous environment at a fixed temperature. This is represented in the model by a thermal bath exerting both a dissipative and a white, Gaussian stochastic force. We believe this does not require further justification.

With the last two ingredients only, namely, with the ratchet potential and the thermal 
bath, the "particle" will obey Levinthal paradox and would not find its way to the native state, particularly in a multidimensional space. The directed transport mechanism requires the presence of a time-dependent external source of energy acting on the particle in order to produce work, in the form of a directional current [25]. To represent a natural, non-designed process, this force must be unbiased. It turns out that the interplay of the asymmetry of the conservative ratchet potential with the external source of energy, yields the sough for directed motion. We shall demonstrate below how the introduction of this force makes the particle finds its way toward the native state and, on the average, in a very short time [13]. The efficacy of the process depends, of course, on the values of the different model parameters.

Regarding the origin of the external energy source, we generally argue that processes in living organisms occur via non-equilibrium states with all sorts of gradients of different physical quantities, and that the maintenance of those gradients may be traced back to the consumption of energy. In a more specific way, during the protein synthesis, the action of the ribosome it is an important influence to the folding process, in the sense that this process is almost concluded in an average size protein by the time the ribosome releases its C-terminal end [26]. Furthermore, ribosomes are recognized complex molecular motors that consume energy in the form of GTP throughout the protein synthesis [27, 28, 29]. Although it seems it does not occur in all folding cases, molecular chaperones may represent another good example of an external "force" in the folding process since they consume ATP while assisting in the process [30, 31, 32, 33, 34]. In a broader sense, we may also argue that of all the many processes occurring in the crowded environment of the cell, some of them consume energy and can be used by the protein during the folding process as the required external force to reach the native state.

The mathematical model is, therefore, the overdamped Langevin equation for a Brownian particle embedded in a thermal bath moving in a multidimensional coordinate system, under the influence of a conservative potential $V(\vec{x})$ and in the presence of a time-dependent external force $F_{i}(t)$,

$$
\gamma \frac{d x_{i}(t)}{d t}=f_{i}(t)-\frac{\partial V(\vec{x})}{\partial x_{i}}+F_{i}(t) .
$$

with $i=1,2, \ldots, d$. $\gamma$ is the friction coefficient of the bath; $f_{i}(t)$ is an stochastic Gaussian 
thermal force, with zero mean and with a white spectrum, namely[19]:

$$
\left\langle f_{i}(t) f_{j}\left(t^{\prime}\right)\right\rangle=2 \gamma k T \delta\left(t-t^{\prime}\right) \delta_{i j}
$$

with $i, j=1,2, \ldots, d$. In this expression $T$ is the temperature and $k$ Boltzmann constant. As mentioned, $\left\langle f_{i}(t)\right\rangle=0$ for all $i$. Thus, $\vec{f}(t)$ represents unbiased white noise. The fact that the thermal force correlation is proportional to $\gamma k T$ is the statement of detailed balance between the thermal bath and the particle [19, 20].

As mentioned, the forces $F_{i}(t)$ represent the action of agents external to the protein itself and the thermal bath, and its driving is caused by ATP consumption. We really do not know the precise form of this force. However, as far as producing directed transport, its precise form is not essential, as long as its correlation time is different from that of the bath [14, 25]. Therefore, we have decided to employ a stochastic force generated by an Ornstein-Uhlenbeck process [19, 20] given by,

$$
\frac{d F_{i}(t)}{d t}=-\frac{F_{i}(t)}{\tau_{e}}+\zeta_{i}(t)
$$

for $i=1, \ldots, d$ and where the functions $\zeta_{i}(t)$ represent independent stochastic variables with zero mean $\left\langle\zeta_{i}(t)\right\rangle=0$, and Gaussian white noise,

$$
\left\langle\zeta_{i}(t) \zeta_{j}\left(t^{\prime}\right)\right\rangle=\frac{f_{o}^{2}}{\tau_{e}^{2}} \delta\left(t-t^{\prime}\right) \delta_{i j}
$$

The parameters $\tau_{e}$ and $f_{o}$ determine the process and represent the correlation time and the fluctuations magnitude of the external force, respectively. As we pointed out above, it has been proved that Brownian motors also work with different kind of zero bias forces, such as sinusoidal or dichotomic ones [15].

\section{RESULTS}

We solve Eqs.(2)-(5) numerically. We first fix the potential parameters, $V_{0}, V_{1}, a, b, c$ and $\lambda$, and then we vary $\gamma, T, f_{o}$ and $\tau_{e}$ such that the directed transport process is optimized. Due to numerical easiness, we study the evolution of a protein with six degrees of freedom. However, to show the robustness of the model we also present results for a protein with 100 degrees of freedom. This may represent a real protein with several tens of aminoacids. 
(a)

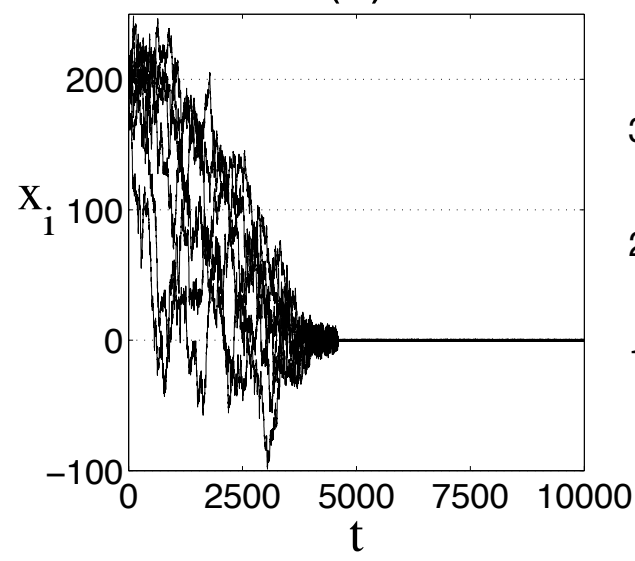

(b)

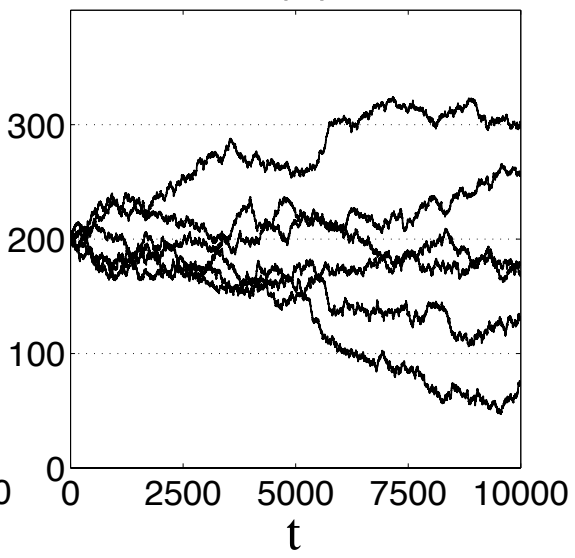

FIG. 2: Typical folding realization of a protein with 6 degrees of freedom. Each line refers to the evolution of a single coordinate $x_{i}$, with $i=1, \ldots, 6$. (a) The stochastic external force is included. (b) No external force is considered and no folding occurs. In this case, $\gamma=1, k T=0.1, f_{0}=3.0$ and $\tau_{e}=0.5$. The initial condition was 200 units in each coordinate for both cases.

\section{A. Folding in 6 dimensions}

Figures $2 \mathrm{a}$ and $2 \mathrm{~b}$ show typical examples of the particle evolution in a 6 dimensional space, by following the value of each of the coordinates $x_{i}$ for $i=1, \ldots, 6$. The behavior when the stochastic external force is present is shown in Fig. 2a, while Fig. 2b corresponds to the case when no external force is considered (and the temperature is increased in order to compensate in magnitude the lack of the external force). An evident directed transport towards the native state is observed in the former case, in contrast with the random-walker behavior when no external force is added. The directed transport occurs (Fig. 2a) until the particle reaches the vicinity of the native state well. Then, a fluctuation takes place and the particle finally falls into the well. If the well is not wide enough the particle may stay for a long time around the minimum of the well. Once the particle is within the well, its position fluctuates depending on, the width of the well at its bottom, the magnitude of the two stochastic forces, and the dimensionality of the space. Typically, for spaces with higher dimensionality wider wells need to be considered in order to make the particle reach the native state. In Fig. 3, we show the evolution of the dimensionless radius position $r^{\prime}=\left(x_{1}^{2}+x_{2}^{2}+\ldots+x_{d}^{2}\right)^{\frac{1}{2}} / r_{o}$, with $r_{o}$ the initial condition. This figure corresponds to typical cases such as those analyzed above as well as the average for 100 realizations. For the 


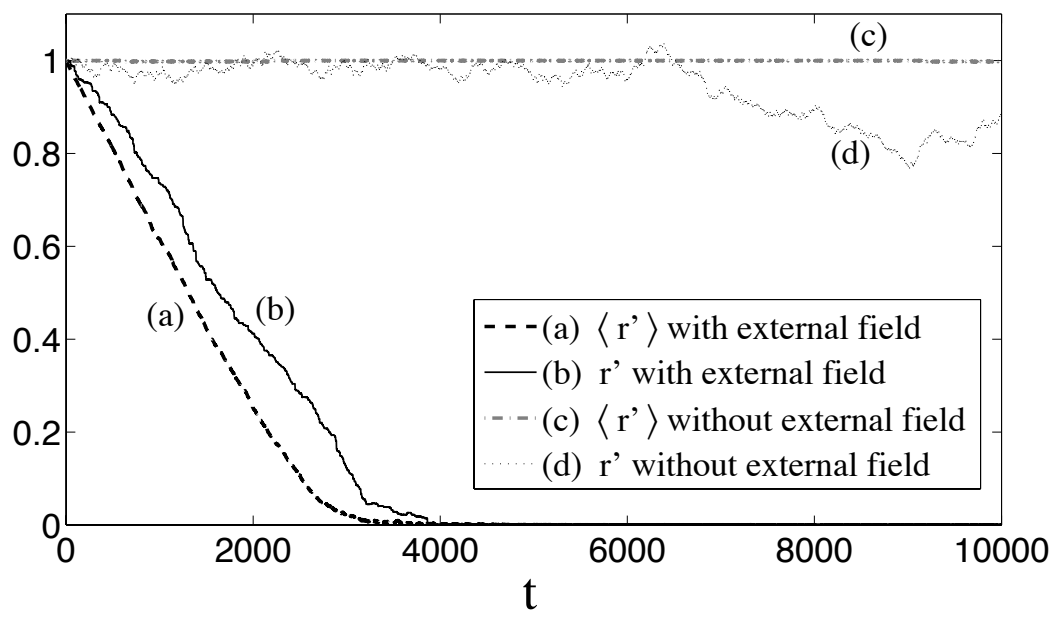

FIG. 3: Time evolution of the dimensionless distance to the native state $r^{\prime}=\left(x_{1}^{2}+x_{2}^{2}+\ldots+x_{d}^{2}\right)^{\frac{1}{2}} / r_{o}$, with $r_{o}$ the initial condition, for a protein with 6 degrees of freedom. Single realizations and averages over 100 realizations are shown for both cases, with and without external forces. Same parameters as in Fig, 2 ,

relevant case when the external force is included, cases (a) and (b) in the figure, we have found $100 \%$ efficacy of "folding" within the arbitrary time limit of 10000 units of time for the parameters considered. This time is very short even in CPU time scales. When the external force is absent, the particle is a true diffusing random walker and, insofar our numerical calculations, we have never seen even a single occurrence of finding the native state. This point serves to emphasize the role of the external force as the responsible one for delivering the energy to be further converted by the ratchet into useful work. The result of the work done is the folding of the protein.

\section{B. Robustness}

In order to demonstrate the robustness of the model two representative cases were studied. The first consisted on increasing the degrees of freedom up to 100 to verify that the dimensionality of the space has no fundamental influence on the behavior of the Brownian motor. The second case considers the inclusion of obstacles into the energy landscape, such as potential barriers and wells, in order to evaluate the capacity of the folding process to overcome such expected features in more realistic scenarios. 


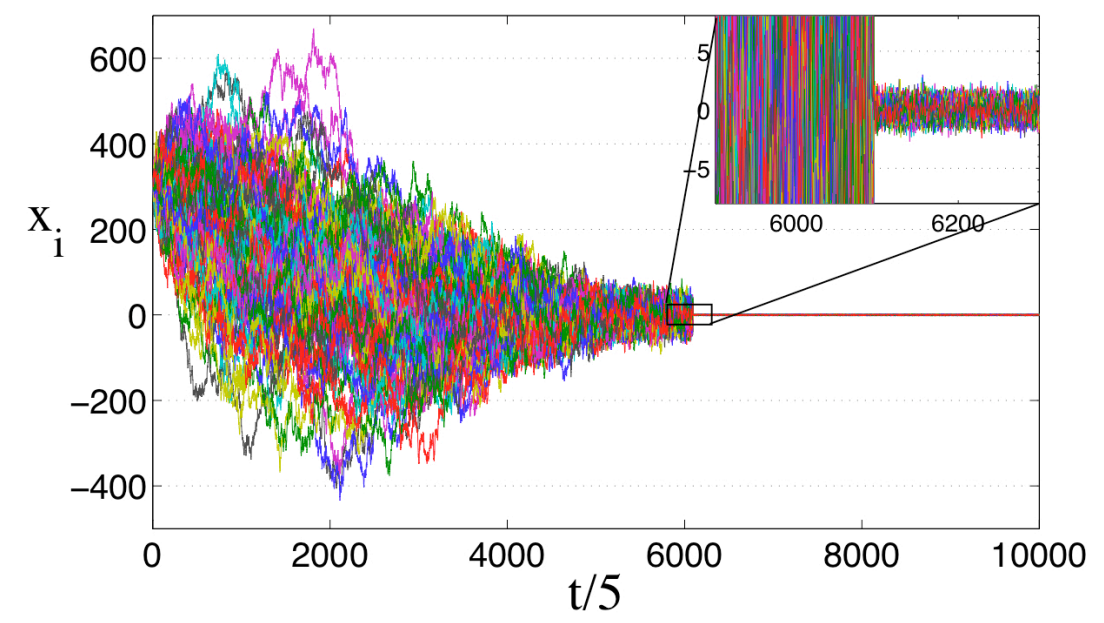

FIG. 4: Typical folding realization of a protein with 100 degrees of freedom, with the external force present. Each color refers to the evolution of a single coordinate $x_{i}$, with $i=1, \ldots, 100$. Here, we used the same potential parameters as in Fig.1 except $V_{1}=10000$ and $c=6000$, while the other parameters are: $\gamma=1, k T=0.1, f_{0}=3.0$ and $\tau_{e}=0.5$. The initial condition is 300 units for each coordinate.

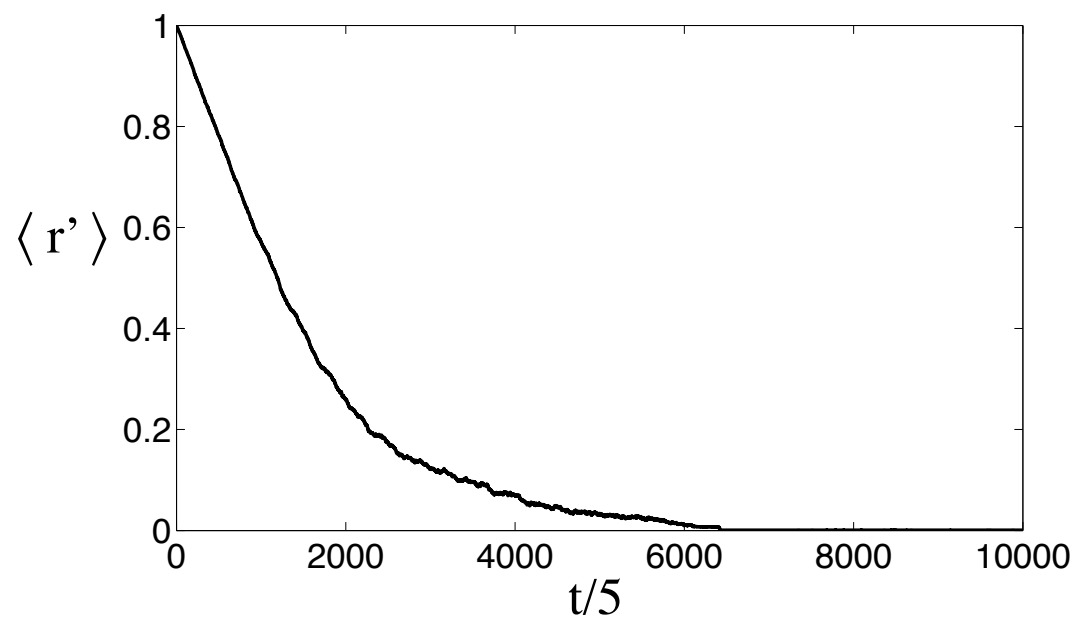

FIG. 5: Time evolution of the dimensionless distance to the native state $r^{\prime}$, see Fig $\sqrt[3]{\text {, averaged }}$ over 20 realizations for a protein with 100 degrees of freedom. Same parameters as in Fig 4 .

\section{100 dimensions}

Figure 4 shows the evolution of the 100 state coordinates of a protein with 100 degrees of freedom. As before, the particle is under the influence of a potential such as that of Eq. (1) and the external field is included. Figure 5 shows the time dependence of the dimensionless 
radius $\left\langle r^{\prime}\right\rangle$, averaged over 20 realizations with the same conditions as the case shown in Fig. 4. In this case, the initial condition is 300 spatial units for each of the coordinates. We find that the overall behavior for 100 coordinates is essentially the same as for the 6dimension case, showing an evident directed transport towards the native state with 100\% of success reaching it. There are, as expected, quantitative differences as the dimensionality is increased. One is the increment in time to reach the native state, as seen in the figures. Other is the increase in size of the spatial fluctuations around the well. Both effects are a consequence of having much more space to explore in the directions orthogonal to the radial one, which is the unique direction where the ratchet asymmetry is present. To be precise, within the parameters used, in order for the particle to get into the native state well, it was necessary to increase the width of the well from 10 to 250 space units as increasing from 6 to 100 dimensions. Further, in order to achieve small fluctuation amplitudes in the native state, see the zoom-in graph in Fig, 4, the width at the bottom of the well was kept narrow but its depth was increased. It should be also clear that the time to reach the native state depends on how far from it the particle is placed initially. At the model level this is a matter of CPU time only.

\section{6 dimensions with obstacles}

The bidimensional representation of the energy landscape of a protein with two additional obstacles is shown in Fig. 6. The obstacles consist on, one, an additional Gaussian well (I) deeper and wider than the native state well (N), (implying in principle a more stable state) and, two, a Gaussian barrier between the two wells and surrounding the native state. The equation corresponding to this potential with obstacles in $d$ dimensions is given by

$$
\begin{array}{r}
V(\vec{x})=V_{0}\left[\sin \left(\frac{2 \pi r}{\lambda}\right)+a \sin \left(\frac{4 \pi r}{\lambda}\right)+b \sin \left(\frac{6 \pi r}{\lambda}\right)\right] \\
-V_{1} e^{-r^{2} / c}-V_{b} e^{-\left(r-r_{b}\right)^{2} / c_{b}}+V_{w} e^{-s_{w}^{2} / c_{w}}
\end{array}
$$

with $s_{w}^{2}=\left(x_{1}-x_{1}^{w}\right)^{2}+\left(x_{2}-x_{2}^{w}\right)^{2}+\cdots+\left(x_{d}-x_{d}^{w}\right)^{2}$. The new parameters $V_{b}, V_{w}, c_{b}$ and $c_{w}$ are the positive real numbers that determine the shape of the barrier and the well introduced, while $r_{b}$ and $\overrightarrow{x_{w}}=\left(x_{1}^{w}, x_{2}^{w}, \ldots, x_{d}^{w}\right)$ determine their positions, respectively.

The behavior of a particle in a 6 dimensional space, under the influence of the potential with obstacles, Eq. (6), and subjected to the stochastic external field, is shown in Fig. 7 . 


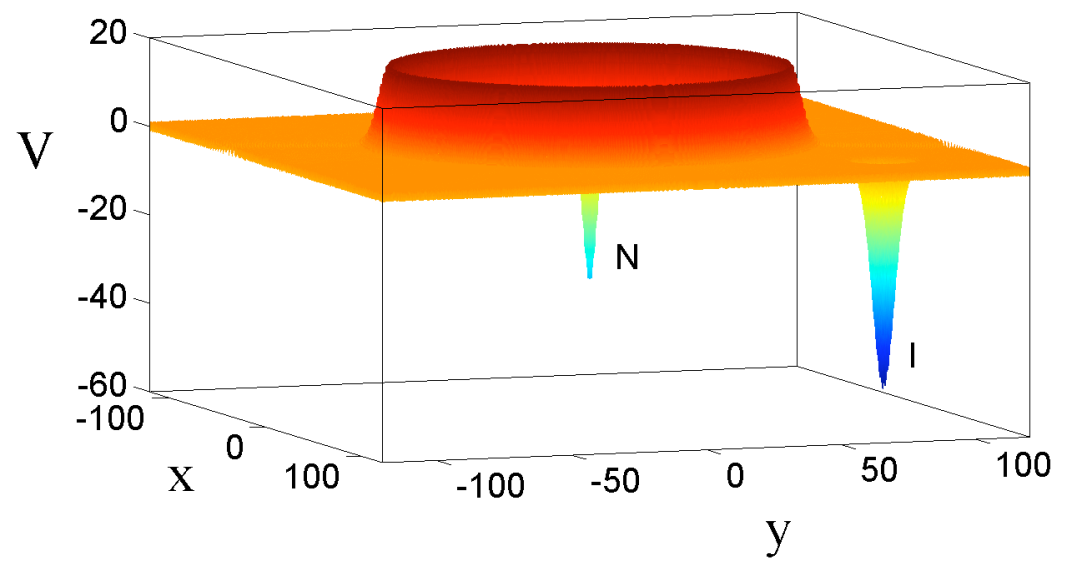

FIG. 6: Bidimensional representation of the potential with obstacles, Eq. 6. Here, $\lambda=1, V_{0}=1$, $V_{1}=50, a=0.4, b=0.1, c=10, V_{b}=18, V_{w}=60, c_{b}=30, c_{w}=30, r_{b}=250$, and $x_{i}^{w}=200$ for $i=1, \ldots, d$. This conservative potential corresponds to the energy landscape of a protein with the native state $(\mathrm{N})$ centered in the origin and two additional obstacles, a well (I) far away from the native state and a barrier between the two wells.

It is important to mention that the initial condition was set at the minimum of the added well (I). Fig. 7 shows that the particle is able to escape from the first, deeper but wider well, then overcome the potential barrier and finally find the native state. Frequently, the obstacles are not overcome immediately, as shown in the radius position evolution of typical realizations denoted by (a), (b), (c) and (d). We see regions of constant distances revealing that the particle takes some time leaving the first well and climbing the barrier. However, the particle always reaches the native state within the arbitrary time limit of 15000 time units, in an average time longer than the case with no obstacles, as the average radius of 100 realizations (e) illustrates.

Therefore, the protein "folds" even if severe obstacles, such as barriers and potential wells, even deeper than that of the native state, are included in the energy landscape. This climbing capacity of the particle is another consequence of the delivery of work by the ratchet. The climbing capability does have a stall limit, thus, that depends on the local slope of the obstacle but not on its height or depth. 


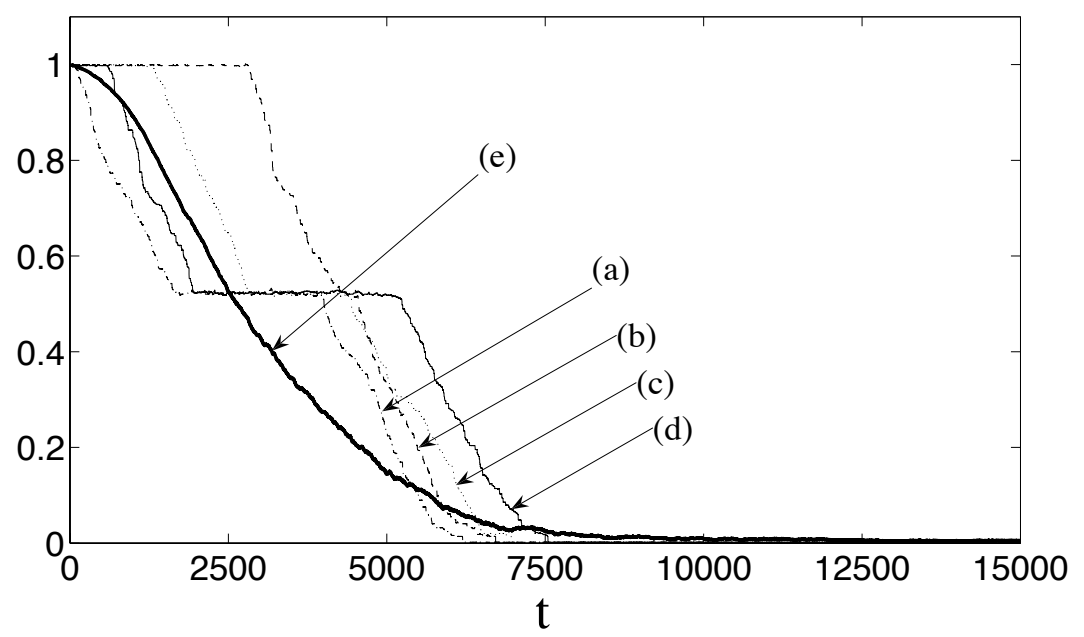

FIG. 7: Time evolution of the dimensionless distance to the native state $r^{\prime}$, see Fig 3 , for a protein with 6 degrees of freedom in the potential with obstacles shown in Fig, 6 . Cases (a)-(d) show typical single realizations and (e) is the average over 100 realizations. The initial state is 200 units in each coordinate and coincides with the minimum of the additional well (I).

\section{CONCLUSIONS}

In this article we have revisited the ratchet or Brownian-motor mechanism extended to several dimensions in order to propose a model for protein folding. The ratchet mechanism has become a potential candidate for many biological processes, since, on the one hand it resembles a thermal engine at a mesoscopic level, but on the other hand, it does not appear to need a specific design such as the man-made engines. That is, it needs only of two essential ingredients: an intrinsic asymmetry and the input of external unbiased energy. The result is delivery of work in the form of directional motion.

With this in mind several authors have used ratchets models in more obvious biological situations in which a transport process, of some kind, is present [15, 16, 17, 18]. Protein folding appears as a process with a directional motion towards the native state in the multidimensional space of the variables that define the energy landscape. These facts persuade us to propose a model for the mechanism of protein folding based on a Brownian motor.

Traditionally, this process has been thought to be driven by free energy differences leading towards a minimum, similarly to a chemical reaction [8], and thus the idea of a funnel in the free energy landscape of the protein is very appealing. The contribution of external agents are welcome in the theory, however, they are not required. Here, by following the 
idea that Life processes consume energy to yield their products, we have speculated that protein folding is driven by an "engine" in which the protein itself is part of it. Therefore, the presence of external sources of energy, provided by the ribosome, molecular choperones or other elements which consume energy during the process, becomes a necessity for the model presented here.

We have focused our work to the in vivo protein folding phenomenon since there are more evident possible sources for the required external energy. However, we think that the model presented can also be considered for the in vitro situation, where part of the bias and external energy necessary to obtain directional work to refold the proteins, may be provided by the laboratory procedures, such as changes of temperature, $\mathrm{pH}$ or concentration of urea [35, 36], among others.

On the other hand, at this moment the most difficult part to justify in our model is the asymmetric structure of the energy landscape. The best that we can say now is that the intrinsic asymmetric structure of proteins, which also presents certain periodicity, encourages us to think that ratchet-like structures can be hidden into the energy landscape. Nevertheless, we do not have evidence neither pro nor con that a ratchet structure is present since this would have to be seen in the highly multidimensional energy landscape.

By means of numerical solutions of the model, it was found that the present model in 6 dimensions is able to produce directed transport in the mentioned space, through which the polypeptide is able to found the native state starting from an arbitrary initial state in the energy landscape and with a 100\% success. This happens when the external source of energy is present, otherwise the finding of the native state is essentially never achieved. When the number of degrees of freedom is extended to 100, again, the process is performed with $100 \%$ efficacy, but due to the larger number of dimensions, the time needed to reach the native state increases approximately 6-fold with the parameters here used. On the other hand, the model demonstrates to be quite robust by showing that the protein is able to overcome severe obstacles in the energy landscape in its way to the target state.

To summarize and to emphasize it again, the interesting and powerful side of this model is that it achieves its goal by taking advantage of two simple requirements only: the intrinsic asymmetric properties of the system and the external energy source. The numerical solutions showed in this work prove that Levinthal's paradox can be solved with a model which does not require ad hoc biased transition probabilities. In contrast, it requires the necessary 
driving of an external energy source, which in our opinion is physically appealing since its presence is essential for Life to occur.

[1] Anfinsen C-B, Haber E, Sela M, White F-H (1961) The kinetics of formation of native ribonuclease during oxidation of the reduced polypeptide chain. Proc Natl Acad Sci USA 47:13091314.

[2] Anfinsen C-B (1973) Principles that govern the folding of protein chains. Science 181:223-230.

[3] Levinthal C (1969) in Mossbauer Spectroscopy in Biological Systems, Proceedings of a meeting held at Allerton House, Monticello, IL, eds DeBrunner and E. Munck (University of Illinois Press), pp 22-24.

[4] Levinthal C (1968) Are there pathways for protein folding? J Chem Phys 65:44-45.

[5] Zwanzig R, Szabo A, Bagchi B (1992) Levinthal's paradox. Proc Natl Acad Sci USA 89:20-22.

[6] Zwanzig R (1995) Simple model of protein folding kinetics. Proc Natl Acad Sci USA 92:98019804 .

[7] Wolynes P-G (2005) Energy landscapes and solved protein-folding problems. Phil Trans $R$ Soc A. 363:453-467.

[8] Bryngelson J-D, Onuchic J-N, Socci N-D, Wolynes P-G (1995) Funnels, pathways and the energy landscape of protein folding. Proteins- Struct., Funct. and Genetics. 21:167-195.

[9] Onuchic J-N, Wolynes P-G (2004) Theory of protein folding. Curr Opin Struct Biol 14:70-75.

[10] Dill K, Bromberg S, Yue K, Fiebig K-M, Yee D-P,Thomas P-D, Chan H-S (1995) Principles of protein folding -A perspective from simple exact models. Protein Sci 4:561-602.

[11] Miller M-A, Wales D-J (1999) Energy Landscape of a Model Protein. J Chem Phys 111:66106616 .

[12] Dill K-A (2008) The protein folding problem. Annu Rev Biophys 37:289-316.

[13] González-Candela E, Romero-Rochín V (2006) Overdamped thermal ratchets in one and more dimensions. Kinesin transport and protein folding. Physica A 372:249-262.

[14] Magnasco M-O (1993) Forced thermal ratchets. Phys Rev Lett 71:1477-1481.

[15] Reimann P. (2001), Brownian motors: noisy transport far from equilibrium. Phys Rep 361:57261. and references therein.

[16] Hanggi P, Marchesoni F, Nori F (2005) Brownian motors. Ann Phys 14:51-70. and references 
therein.

[17] Bartussek R, Reimann P, Hanggi P (1996) Precise numerics versus theory for correlation ratchets. Phys Rev Lett 76:1166-1169.

[18] Astumian R-D, Hanggi P (2002) Brownian motors. Phys. Today 55:33-39.

[19] van Kampen N-G (1992) Stochastiic Processes in Physics snd Chemistry. eds Elsevier Science Publishers B.V. (North Holland, Amsterdam) pp 219-220.

[20] Risken H (1984) in The Fokker-Planck equation, eds (Springer, Berlin) pp.

[21] Feynman R-P, Leighton R-B, Sands M (1963) The Feynman Lectures on Physics, Vol. I, (Addison Wesley, Reading Massachusets) pp 46.1-46.9.

[22] Doering C-R, Horsthemke W, Riordan J (1994) Nonequilibrium fluctuation-induced transport. Phys. Rev. Lett. 72:2984-2987.

[23] Astumian R-D, Bier M (1994) Fluctuation driven ratchets: Molecular motors. Phys. Rev. Lett. 72:1766-1769.

[24] The multidimensional free energy landscape may already have the information that the system is immersed in water. That is, the interaction potential should have all effective interactions due to the hydrophilic or hydrophobic properties of the different aminoacids.

[25] Ibarra-Bracamontes L, Romero-Rochín V (1997) Stochastic ratchets with colored thermal noise. Phys. Rev. E 56:4048-4051.

[26] Alberts, A. Johnson, J. Lewis, M. Raff, K. Roberts, and P. Walter, Molecular biology of the cell, Garland, New York, (2002).

[27] Sinha D-K, Bhalla U-S, Shivashankar G-V (2004) Kinetic measurement of ribosome motor stalling force. AppPhysLett J. 85:4789-4791.

[28] Visscher K (2009) Motion of individual ribosomes along mRNA. Proccedings of the American Physical Society, 71st Annual Meeting of the Southeastern Section, 11-13 November, 2004, Oak Ridge, TN.

[29] Garai A, Chowdhury D, Chowdhury D, Ramakrishnan T-V (2009) Stochastic kinetics of ribosomes: single motor properties and collective behavior. Phys Rev E 80:011908-011922.

[30] Ellis R-J, Hartl F-U (1996) Protein folding in the cell: competing models of chaperonin function. FASEB J. 10:20-26.

[31] Hartl F-U (2002) Molecular chaperones in the cytosol: from nascent chain to folded protein. Science 295:1852-1858. 
[32] Bhutani N, Udgaonkar J-B, (1994) Chaperonins as protein-folding machines. Current Science $83: 1337-1351$.

[33] Walter S, Buchner J (2002) Molecular chaperones-cellular machines for protein folding. Ange. Chem. Int. Ed. 41:1098-1113.

[34] Goloubinoff P, Christeller J-T, Gatenby A-A, Lorimer G-H (1989) Reconstitution of active dimeric ribulose bisphosphate carboxylase from an unfolded state depends on two chaperonin proteins and Mg-ATP. Nature 342:884-889.

[35] Lapanje S Physicochemical aspects of protein denaturation, Wiley, New York, (1978).

[36] Auton M, Holthuzen L-M, Bolen D-W (2007) Anatomy of energetic changes accompanying urea induced protein denaturation. from an unfolded state depends on two chaperonin proteins and Mg-ATP. Proc Natl Acad Sci USA 104:15317-22. 